\title{
Matching Theory of Cross-Polarization Suppression Components for Large-Scale Rectangular Waveguide Slot Array Antennas
}

\author{
Boyuan $\mathrm{Ma}^{1, \mathrm{a}, *}$ and $\mathrm{J}$. Pan ${ }^{1, \mathrm{~b}}$ \\ ${ }^{1}$ School of Electronic Engineering, University of Electronic Science and Technology of China, Chengdu, \\ China \\ ${ }^{2}$ School of Electronic Engineering, University of Electronic Science and Technology of China, Chengdu, \\ China \\ a 331953406@qq.com, banjin@uestc.edu.cn
}

Keywords: slot array antenna; cross-polarization suppression; parallel metallic plates

\begin{abstract}
In order to obtain good circular polarization, parallel metallic plates are used for large-scale rectangular waveguide slot array antennas to achieve pure linear polarization at first. It is found that these plates not only suppress cross-polarization, but also influence the original amplitude distribution of arrays for co-polarization. Though it is easy to obtain the proper plates length to achieve satisfying cross-polarization suppression. Plates with a specific length drastically perturb the array distribution, which leads to severe degradation of radiation performance. A matching theory for plates design is proposed and verified. It provides proper parameters for parallel metallic plates, and criteria for judging the design results. A practical array antenna is fabricated. Both the simulation and measurement results validate this theory. The theory gives guidance of designing similar external components for cross-polarization suppression or other functions, which is useful for slot arrays and other array antennas.
\end{abstract}

\section{Introduction}

Large-scale rectangular waveguide slot array antennas (LSRWSAAs) have long been used as surface monitor radar, ship radar, port radar etc. They can provide high gain, low side lobe and narrow beam width with relatively compact size, simple configuration and well stability. Fine bandwidth can also be achieved by employing traveling-wave arrays ${ }^{[1]}$.

Circular polarization is one of the common requirements of modern radar systems. In order to meet this requirement, LSRWSAAs, with consideration for design and fabrication simplicity, generally need to achieve great cross-polarization suppression at first ${ }^{[2]}$. Several simple methods have been studied to suppress cross-polarization ${ }^{[1,3-6]}$, such as choke slots, metallic grids, adjusting the distance between antennas and ground plane, parallel metallic plates etc. Choke slots are not preferred for large-scale arrays because of their big volume, heavy weight and narrow bandwidth. Metallic grids are difficult to install and stablish, especially when antennas need to rotate. Adjusting the distance between the antenna and ground plane obvious brings about extra volume and weight. More complicated methods like untilted slots and irregular structure are also studied, but they brings about much design complexity ${ }^{[7,8]}$. Parallel metallic plates are simple, small, cheap, stable, and wide-band, which makes them quite useful for LSRWSAAs. However, it is found that parallel metallic plates affect the designed amplitude distribution of the original slot array. They influence the matching of each slot so that the radiation performance degrades. These side effects can be avoided by properly adjusting the plate parameter. However, parameter adjustment is tough for large-scale arrays, especially when a horn is needed at the broadside of arrays to enhance the radiation performance. In this article, a matching theory is introduced to adjust the plate structure to obtain satisfying cross-polarization suppression, distribution invariance and radiation performance. An X-band narrow-wall LSRWSAA which employs Taylor distribution with 266 slots and a length of $6.6 \mathrm{~m}$ is simulated, fabricated, and measured. Both the simulation and measurement results validate the theory. 


\section{Parallel Metallic Plates}

A narrow-wall slot array shown in Figure 1 employs horizontal polarization as co-polarization. Parallel metallic plates, with or without a horn, utilize cut-off modes of H-plane horns or plane waveguides respectively to suppress cross-polarization. The horizontally-polarized wave propagates as the TEM or $\mathrm{TE}_{10}$ mode of the plane waveguide or horn respectively. Meanwhile, the vertically-polarized wave propagates as a cut-off mode. As a result, cross-polarization is suppressed.

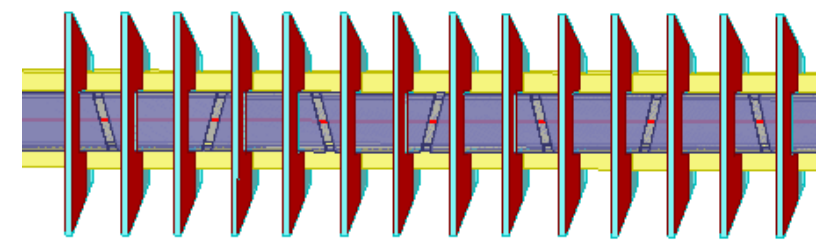

Figure 1 Narrow-wall slot array with parallel metallic plate.

\subsection{Design Method}

A slot array in Figure 1 utilizes different tilts angle and incision depths of each slot to design the amplitude distribution along the array ${ }^{[6]}$. Because parallel metallic plates change the original amplitude distribution, their structural parameters have to be decided before designing slots. Then, the plates need to be considered as a part of the waveguide during slot design. If a horn is needed, a partial horn should also be under consideration.

\subsection{Structure}

As shown in Figure 2, for ease of comparing the models with and without a horn, trapezoidal parallel metallic plates are employed. The functional part of the plates is mainly defined by $f l$, and $\theta$ defines angle of elevation of the plates and horn. There is a pair of plates for each slot. The plates and the waveguide are locked together for stability. A horn is added like Figure 3. In the following part, parallel metallic plates without a horn are called an unconfined model, and those with a horn are called a confined model. The fabricated antenna is shown in Figure 4 and 5 . The array antenna has 266 slots, 532 plates and a length of $6.6 \mathrm{~m}$.

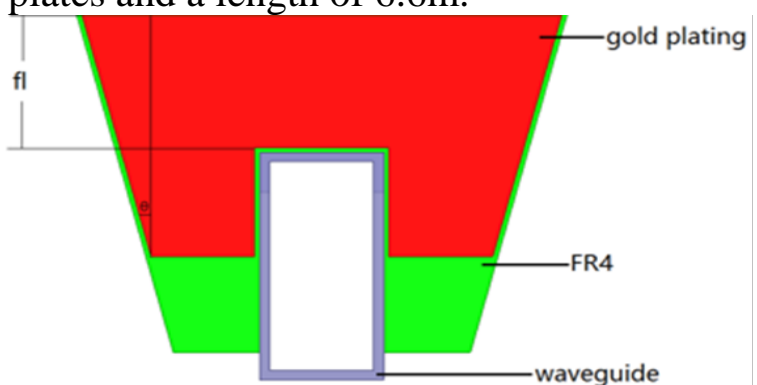

Figure 2 Parallel metallic plates

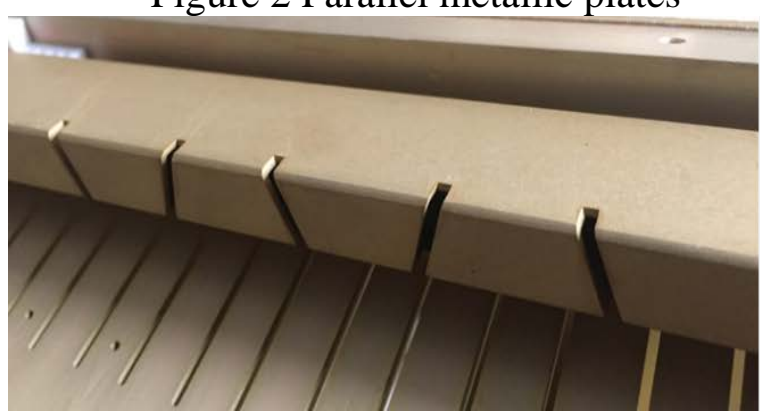

Figure 4 Fabricated slot array

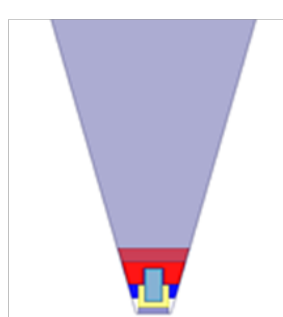

Figure 3 Adding a horn

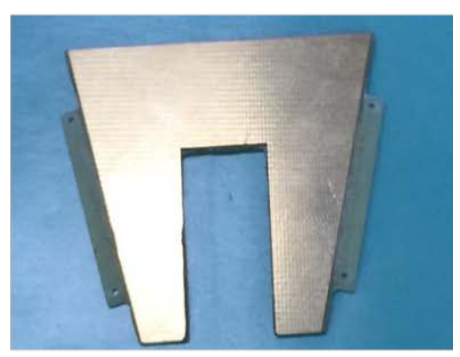

Figure 5 Fabricated parallel metallic plates 


\subsection{Unconfined Model}

In an unconfined model, the plates function like plane waveguides. As shown in Figure 6, in a classical plane waveguide with proper structure parameters, the horizontally-polarized wave propagates as the TEM mode, while the vertically-polarized wave propagates as cut-off TE modes ${ }^{[9]}$. The width of the practical plates is narrow, especially at the start edge. Besides, they only have a limited length and are excited by slots rather than waveguides or incident plane waves at the start ports. These make the plates unconfined non-classical plane waveguides, which means partial incident waves does not propagate along the waveguide and the propagating waves do not completely behave like typical modes. Though rigorous analytical results are not precise any more, the classical theory is still useful. As plane waveguides, the plates need enough length to suppress cross-polarization and proper length to avoid affecting co-polarization. The propagation constant $\gamma$ of the cross-polarized wave can be calculated by ${ }^{[9]}$

$$
\gamma^{2}=k_{c}^{2}-k^{2}
$$

where $k_{c}$ and $k$ is the cut-off wavenumber and wavenumber in homogenous medium respectively. Because the amplitude of the cross-polarization varies as $e^{-\gamma l}$, it decays quickly as shown in Figure 7. The length of the plates for cross-polarization suppression can be easily calculated by Equation (1), or obtained by simple single waveguide simulation like Figure 7. Since the length for cross-polarization suppression is easy to obtain, invariance of designed amplitude distribution, i.e. co-polarization matching is important. Because the co-polarized wave propagates as the TEM mode in plane waveguides, a pair of parallel metallic plates have similar characteristics with an open-ended transmission lines ${ }^{[9]}$ in Figure 8.

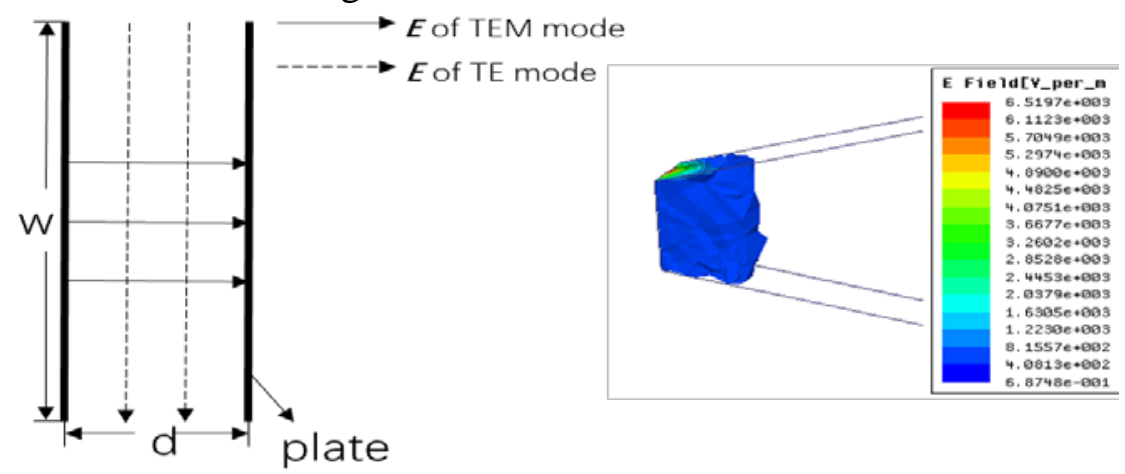

Figure 6 Modes of a plane waveguide

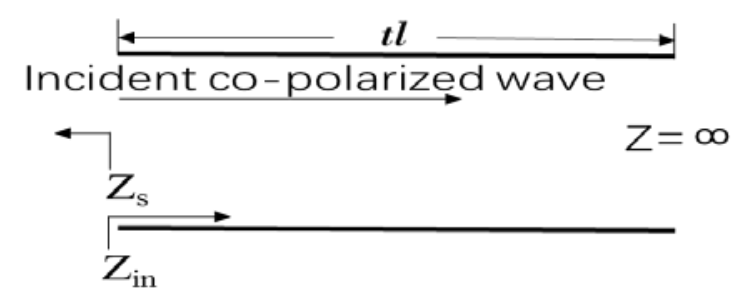

Figure 7 Cross-polarization in a plane waveguide

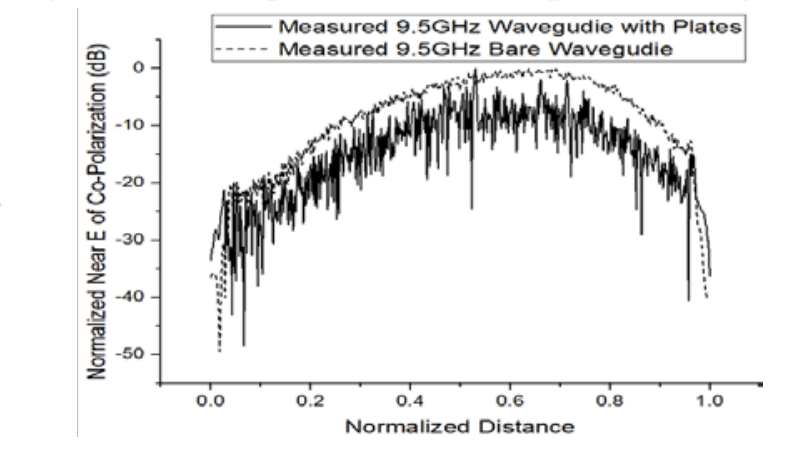

Figure 9 Normalized Measured Near Field of Co-Polarization at $9.5 \mathrm{GHz}$
Figure 8 Approximate transmission line model model of a pair of plates Because a slot array in Figure 1 needs different slot conductance for each slot to achieve specific
mplitude distribution, $Z_{s}$ of each pair of plates is different, which makes it difficult to match every amplitude distribution, $Z_{s}$ of each pair of plates is different, which makes it dificult to mater conery
slot and its plates. Given consideration to design and fabrication complexity for different pairs of plates is not acceptable. However, overall matching design is still necessary and feasible. For an open-ended transmission line in Figure 8, $Z_{\text {in }}$ equals infinity when its length $t l$ equals half the wavelength of the incident wave, where one of the worst matching conditions is 
obtained $^{[9]}$. In this case, designed amplitude distribution should be significantly influenced. However, because of the unique incision depths of each slot in Figure 4, $t$ is larger than the plate length $f l$ shown in Figure 2. In order to verify the theory, a simulation model was designed, and a practical array was designed, fabricated and measured with $f l$ being $15 \mathrm{~mm}$. This length does not include the different incision depths of each slot, because they cannot be directly added to $\mathrm{fl}$.

For the measured results, both the worst near-field and far-field results of co-polarization are at 9.5GHz, as shown in Figure 9-11. At frequencies away from $9.5 \mathrm{GHz}$, the near field is smoother and varies less with frequency. At $9.5 \mathrm{GHz}$, though adding plates along the waveguide has to change the amplitude distribution, these significant changes in terms of overall tendency and smoothness lead to decreasing gain and poor SLL. The measured far-field results are shown in Figure 11. It is quite obvious that the far-field pattern of $9.5 \mathrm{GHz}$ has much worse SLL. For simulation results, the gain and SLL of the far field are shown and compared in Table 1. It is found that the worst case of the simulation and measurement results are around $9.3 \mathrm{GHz}$ and $9.5 \mathrm{GHz}$ respectively, and the designed plate length is half the wavelength at $10 \mathrm{GHz}$. This could be caused by the following reasons. First, the slots have incision depth at the wide wall, as shown in Figure 4, which implies that the actual length of the waveguide is longer than the design length $f l$ in Figure 2. As a result, the frequency of the worst case should be lower. Next, these non-classical unconfined plane waveguides are short, narrow, and trapezoidal. Besides, they are excited by slots instead of waveguides or incident waves. And each slot has different structure to obtain different radiation conductance, so the worst case should happen at the frequency where the overall matching is the poorest. For the difference between simulation and measurement, machining errors and abrasion are not avoidable for practical plates. A difference of $2.13 \%$ between simulation and measurement is quite acceptable. From these results, it is inferred that length of parallel metallic plates influences the original amplitude distribution of the array, and when the length is around half the wavelength, the worst matching condition is obtained. Besides, the ill-condition bandwidth of a specific plate length is quite narrow like HI-Q components, which means the function of the plates is sensitive to operating frequency and plate length. Such ill conditions can be avoided by carefully designing structure of the plates based on operating frequency.

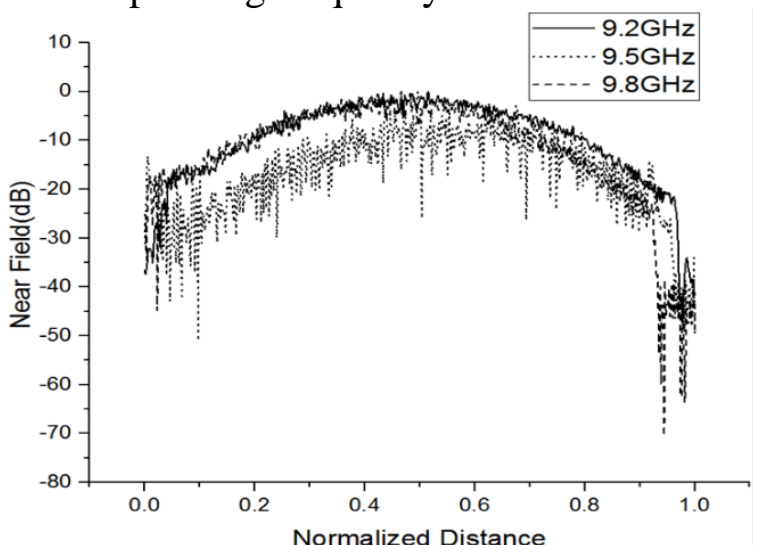

Figure 10 Normalized Measured Near Field of Co-Polarization

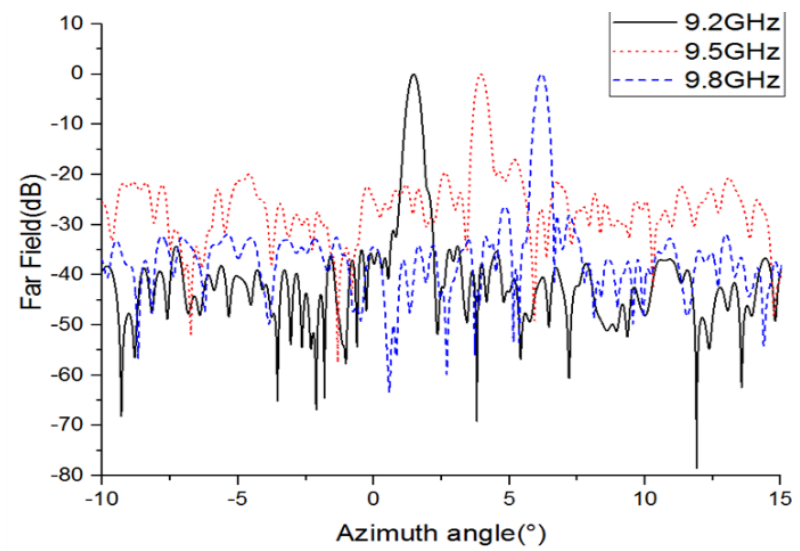

Figure 11 Normalized Measured Far Field of Co-Polarization

Table 1 Simulation Gain and SLL at Different Frequencies.

\begin{tabular}{|c|c|c|c|}
\hline Frequency(GHz) & Gain(dB) & SLL(dB) & Radiation Efficiency \\
\hline 9.18 & 23.3 & -23.6 & 0.52 \\
\hline 9.26 & 17.3 & -16.4 & 0.21 \\
\hline 9.34 & 20.1 & -14.7 & 0.24 \\
\hline 9.42 & 25.1 & -19.3 & 0.49 \\
\hline
\end{tabular}

\subsection{Confined Model}

The same methods were used to analyse the confined model. Similar results were obtained, except that parallel metallic plates with a horn form horn waveguides instead of plane waveguides. 
The propagation wavelengths are different, which leads to the different worst cases in terms of frequency.

\section{Matching Theory}

Based on the above results and analysis, since there is mature theory for cross-polarization suppression ${ }^{[1-6,9]}$, the key point of designing parallel metallic plates is not to affect the original amplitude distribution for co-polarization. Plate lengths around half the wavelength obviously become forbidden choices. Therefore, plate length longer or shorter than this range can be optimal choices. An unconfined model, for example, should employs equivalent plate length of about $\lambda / 8$ longer or shorter than half the wavelength. Given consideration to cost, especially when gold is used, shorter length is better. To reduce the effect of machining errors, longer length prevails. Designers can make choices based on practical situation. Simulation model with longer or shorter plate length are given in the following. An undefined model is applied. The chose length ranges from $11 \mathrm{~mm}$ to $19 \mathrm{~mm}$, and the better results appears at $11 \mathrm{~mm}$ and $19 \mathrm{~mm}$ as excepted. The near field of co-polarization in ill and good conditions are shown in Figure 12. A good-condition near field looks much smoother and varies less with frequency than an ill-condition near field does. Besides, a good-condition near field are always related to good far field performance, because it affects the distribution much less. Minimal co-polarization and maximal cross-polarization at 9.18-9.42GHz are shown in Figure 13. The results at both edges are better, which validates the theory. This theory is also feasible to a confined model.

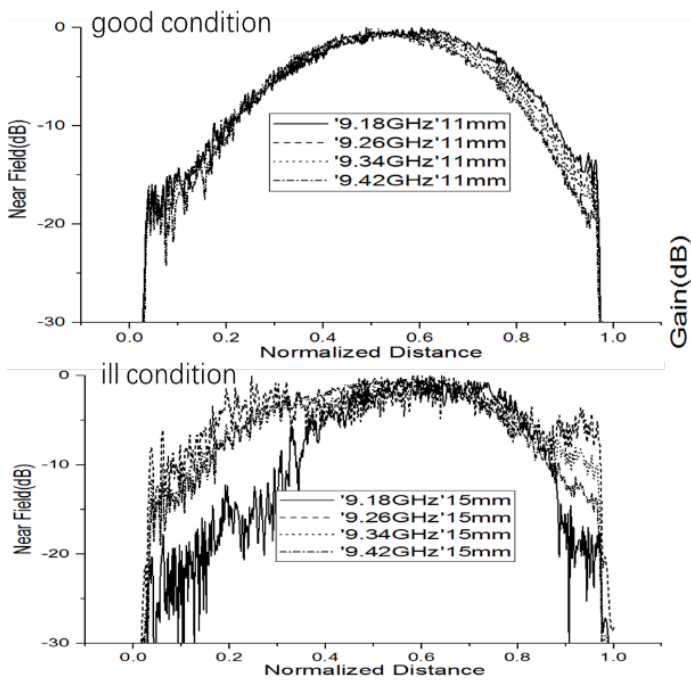

Figure 12 Near Field of Co-Polarization

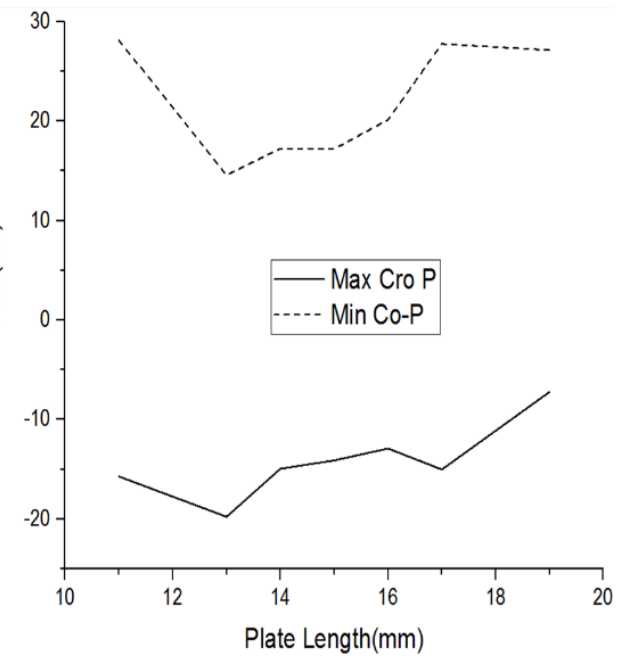

Figure 13 Gain of each polarization

\section{Conclusion}

In order to suppress cross-polarization and protect co-polarization, the optimal length of parallel metallic plates for LSRWSAAs is studied. It shows that cross-polarization suppression is simple for this structure, but it introduces co-polarization mismatch when the plate length is around half the wavelength. An approximate transmission line model is used to demonstrate its mechanism, and both simulation and measurement results validate the theory. Based on these, a matching theory for plate length is presented and verified. Simulation results show that the plate length should be shorter or longer than the range around half the wavelength. The results also give criteria in terms of near and far fields to decide if the length is proper. This theory is quite useful for similar arrays and gives guidance for other polarization suppression components. Further research can study the analytical reflection efficient of the waveguides by separate inward and outward propagating waves, and then gives the precise forbidden length choices. Extracting precise equivalent RLC parameters for a complete transmission line model is also practicable. The different incision depths for each slot could also be considered by adding an equivalent length to the plate length. 


\section{References}

[1] G. A. Xiong, J. Pan, et al. Low Cross-Polarization Slab Waveguide Filter for Narrow-Wall Slotted Waveguide Array Antenna with High Gain Horn[C]. (2016) International Conference on Mechatronics Engineering and Information Technology, 266-270.

[2] Min K. S, et al. A Circular Polarized Waveguide Narrow-Wall Slot Array Using a Single Lauer Polarization Inverter[C]. (1996) Antennas and Propagation Society International Symposium, 1004-1007

[3] J. Z. Nan, J. Pan, et al. A structure of Cross-polarization Inhibition for Narrow Wall Slot Waveguide Antenna[C]. (2016) International Conference on Advances in Energy, Environment and Chemical Engineering, 329-334

[4] M. Mazur, J. Wisniewski. Performance of Cross-Polarization Filter Dedicated for Slotted Waveguide Array Antenna[C]. (2010) IEEE Radar Conference, 1335-1338

[5] A.Mallahzadeh, S. Mohammadalinezhad. An Ultralow Cross-Polarization Slotted Waveguide Chebyshev Array Antenna[C]. (2011) European Conference on Antennas and Propagation, 1953-1956

[6] G. A. Xiong, J. Pan, et al. Narrow-wall Slotted Waveguide Array Antenna with Low Cross-Polarization Filter[C]. (2016) International Conference on Machinery, Materials, Environment, Biotechnology and Computer, 705-709

[7] Moradian M. Untilted Edge-Slot Antenna Arrays Fed by Wiggly Double-Ridged Waveguide[J]. (2015) Electronics Letters, 51(24), 1966-1967

[8] A. R Mallahzadeh, S. M. A. Nezhad. (2012) Cross-Polarization and Size Reduction of Slotted Waveguide Array by Angled Ridges[C]. International Conference on Machinery, Materials, Environment, Biotechnology and Computer, 705-709

[9] David M. Pozar. Microwave Engineering, Third Edition [M]. (2005) John Wiley \& Sons, Inc. ISBN 0-471-44878-8, 49-55, 84-90 